\title{
Retinal nerve fibre layer polarimetry: histological and clinical comparison
}

\author{
J E Morgan, A Waldock, G Jeffery, A Cowey
}

Cardiff Eye Unit, University Hospital of Wales, Cardiff CF4 4XW

J E Morgan

Bristol Eye Hospital, Lower Maudlin Street, Bristol BS1 2LX A Waldock

Institute of Ophthalmology, Bath Street, London EC1 2PD

G Jeffery

Department of Psychology, South Parks Road, Oxford OX1 3PT

A Cowey

Correspondence to: Mr J E Morgan, Cardiff Eye Unit, University Hospital of Wales, Heath Park Cardiff CF4 4XW.

Accepted for publication 9 January 1998

\begin{abstract}
Aims-To compare histological thickness of the retinal nerve fibre layer in the primate with retardation measurements obtained in vivo using the Mark II Nerve Fiber Analyzer (NFA, Laser Diagnostic Technologies, San Diego, USA).

Methods-Scanning laser polarimetry was performed on both eyes of a healthy anaesthetised adult primate (Macaca mulatta). The retinal nerve fibre layer thickness was measured in the eye with the best polarimetry image. A nerve fibre layer thickness map was scaled and aligned to a retardation map to permit correlation of retardation and thickness measurements. Results-Retinal nerve fibre layer thickness measurements could be satisfactorily aligned with corresponding retardation values at 216 locations. The overall correlation coefficient for nerve fibre layer thickness and retardation was $r=0.70$ ( $\mathrm{n}=216, \mathrm{p}<0.001)$. Regional comparison showed the best correlation $(r=0.76$, $\mathrm{n}=45, \mathrm{p}<0.001)$ occurred inferior to the optic disc. Less positive but still highly significant correlations were seen superiorly and temporally $(r=0.52, \mathrm{n}=26$, $\mathrm{p}=0.007$ and $r=0.49, \mathrm{n}=86, \mathrm{p}=<0.001$ respectively), with the lowest correlation occurring at the nasal aspect of the disc $(r=0.06, \mathrm{n}=67, \mathrm{p}=0.64)$.
\end{abstract}

Conclusions-In the primate eye, retinal nerve fibre layer thickness shows a positive correlation with retardation measurements obtained with the nerve fibre analyser. However, since the correlation coefficient varied around the optic disc, further evaluation of the device is advised before its routine clinical use.

(Br f Ophthalmol 1998;82:684-690)

In glaucoma, retinal ganglion cell death results in axon loss and thinning of the retinal nerve fibre layer. Cell loss may precede visual field deterioration $^{12}$ and it has been estimated that the retinal ganglion cell population at any retinal locus may be reduced by $50 \%$ before this can be detected using conventional perimetric techniques. ${ }^{3}$ Early detection of nerve fibre layer thinning should facilitate the earlier diagnosis of glaucoma and improve the long term prognosis for the patient.

Clinical examination of the retinal nerve fibre layer is valuable in diagnosing disease ${ }^{14}$ but remains, essentially, a subjective technique in spite of the development of several quantification systems..$^{5-7}$ Confocal scanning laser tomography can provide detailed topographic maps of the retinal surface ${ }^{89}$ but requires the derivation of a stable reference plane relative to which changes in retinal surface height can be measured. Currently, reference planes are derived from points on the retinal surface ${ }^{8}$ whose surface height can change with disease progression, thereby reducing sensitivity for the detection of nerve fibre layer thinning.

Scanning laser polarimetry has recently been introduced as a method that can avoid these shortcomings since it has the potential to measure directly the thickness of the retinal nerve fibre layer based on its birefringent properties. ${ }^{10}{ }^{11}$ The retinal nerve fibre layer is thought to behave as a form birefringent medium $^{12}$ as a result of the ordered arrangement of axonal microtubules and neurofilaments. ${ }^{13}$ Previous histological analysis in the primate has shown a clinically useful correlation between nerve fibre layer thickness and retardation measurements. ${ }^{14}$

A complicating factor in these studies has been the effect of birefringent ocular structures such as the cornea ${ }^{15}$ that may adversely influence the correlation of retardation with retinal nerve fibre layer thickness. The Nerve Fiber Analyzer (NFA, Laser Diagnostics Inc, San Diego, USA) is designed to minimise this effect using a proprietary "compensator" which corrects for corneal birefringence. As yet, however, no study has assessed the influence of this device on the correlation of retinal nerve fibre layer birefringence and thickness. In the present study retinal retardation values have been correlated with histological measures of nerve fibre layer thickness in an intact eye. Polarimetric images obtained in the primate are also compared with those obtained from patients examined in the clinical setting.

\section{Methods}

Retardation measurements were made in a single adult male primate (Macaca mulatta). Animal procedures were conducted within ARVO guidelines for the Use of Animals in Ophthalmic and Vision research. The primate weighed $9.6 \mathrm{~kg}$, had taken part in a breeding programme, and was unsuitable for long term experiments. Polarimetric measurements were made 24 hours following recovery from a procedure in which the neuroanatomical tracer, horseradish peroxidase, had been implanted in the visual cortex. The animal was sedated with $20 \mathrm{mg} / \mathrm{kg}$ of ketamine hydrochloride intramuscularly (Vetalar, Parke Davis Veterinary) and then anaesthetised with intravenous sodium thiopentone (Rhone-Merieux). Measurements took about 2 hours during which core temperature, respiratory rate, and anaesthetic 

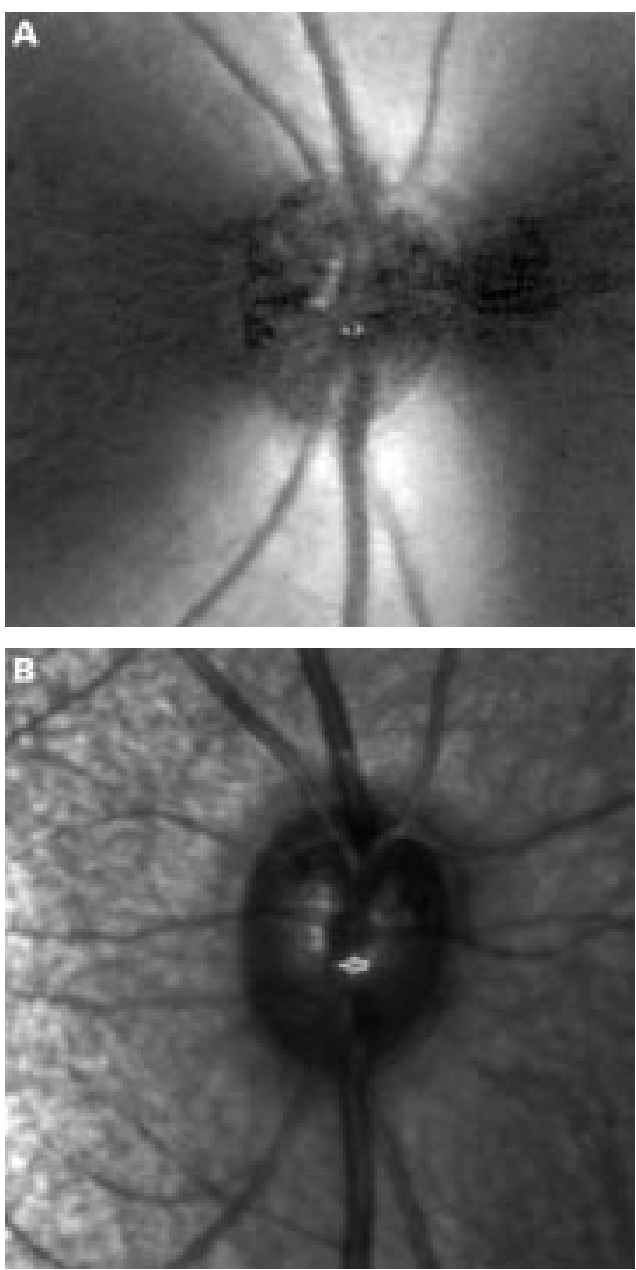

Figure 1 (A) Image of the optic disc of the right eye showing peripapillary birefringence. In the grey scale view, lighter pixels correspond to areas of higher retardation. (B) Extended focus image of the optic disc obtained at the time of the polarimetric scan. The bright spot just inferior to the centre of the optic disc is an imaging artefact.

requirement were continuously monitored. The eyelids of the scanned eye were retracted using 6-0 silk sutures and the corneal surface protected by the liberal application of $0.9 \%$ saline. The retraction suture was released in the periods between examinations to preserve the optical quality of the cornea.

Polarimetric images were taken using a Mark II Nerve Fiber Analyzer (NFA). The technical details of the NFA have been described elsewhere. ${ }^{101116}$ The corneal birefringence compensation range of the NFA was modified by the manufacturer to cope with the polarising effect of the primate cornea. The software permitted simultaneous fundus examination using an extended focus image obtained from the scanning laser ophthalmoscope. An experienced operator obtained at least three images from each eye using the 15 degree field of view and then selected the highest quality image for analysis, being masked to the process of histological reconstruction.

After completion of the polarimetric measurements, the animal was given a further, lethal dose of anaesthetic and the upper body was immediately perfused transcardially with 1 litre of $0.9 \%$ saline at room temperature, followed by 1 litre of fresh $4 \%$ paraformaldehyde in 0.1 $\mathrm{M}$ phosphate buffer ( $\mathrm{pH} 7.4$ ). The eyes were removed intact and postfixed for a further 72 hours in $4 \%$ paraformaldehyde. In the eye selected for comparative measurements, an area of retina and underlying choroid and sclera (approximately $1 \mathrm{~cm}$ square and centred in the optic disc) was removed and fixed for a further 24 hours at room temperature in 30\% sucrose in phosphate buffered $4 \%$ paraformaldehyde. Frozen sections were cut at a thickness of $60 \mu \mathrm{m}$, every section being stored in paraformaldehyde in $0.1 \mathrm{M}$ phosphate buffer at $4^{\circ} \mathrm{C}$. The tissue was oriented so that the sections ran parallel to a line joining the fovea and centre of the optic disc.

Sections were coverslipped under phosphate buffer and examined at $\times 400$ magnification using differential interference contrast (DIC) optics on an Olympus $\mathrm{BH} 2$ microscope. The retinal nerve fibre layer thickness was measured at $360 \mu \mathrm{m}$ intervals along every sixth section using an eyepiece mounted micrometer (2 $\mu \mathrm{m}$ per division). The position of blood vessels and disc margins were noted in these and additional sections to refine the location of these retinal landmarks. A map of retinal nerve fibre layer thickness was constructed using these measurements. Manipulation of the retardation map was facilitated by condensing the 256 $\times 256$ pixel image displayed by the NFA software to one in which each retardation point represented the mean of a $2 \times 2$ pixel square. The thickness values from the NFA were each divided by 7.4 to convert to degrees of retardation. ${ }^{14}{ }^{17}$ Vertical and horizontal scaling for both maps was calculated from the horizontal and vertical dimensions of the optic disc with reference to the extended focus and retardation images. A tracing of the scaled nerve fibre layer thickness map was then placed over the retardation map and final alignment achieved manually, using the optic disc and retinal vasculature as landmarks. Since each section was $60 \mu \mathrm{m}$ thick, the nerve fibre layer thickness measurement was taken to correspond with a $60 \mu \mathrm{m}$ sided square at the retinal surface. The mean of the retardation values that fell within this square was paired with the thickness measurement and used to calculate the correlation coefficient. Samples that bordered on, or overlaid, blood vessels were excluded from the analysis. Statistical analysis was undertaken using SPSS for Windows (Version 6.0, Chicago, IL, USA). Correlation coefficients were calculated using the Pearson correlation coefficient with a two tailed test for significance.

In patients, images were acquired in the course of routine clinical management using the Mark I NFA. All subjects gave informed consent and the study was conducted in accordance within the guideline of the ethical standards committee of the United Bristol HealthCare Trust. At least three scans were performed on each patient for which the best quality image was included in the analysis. 

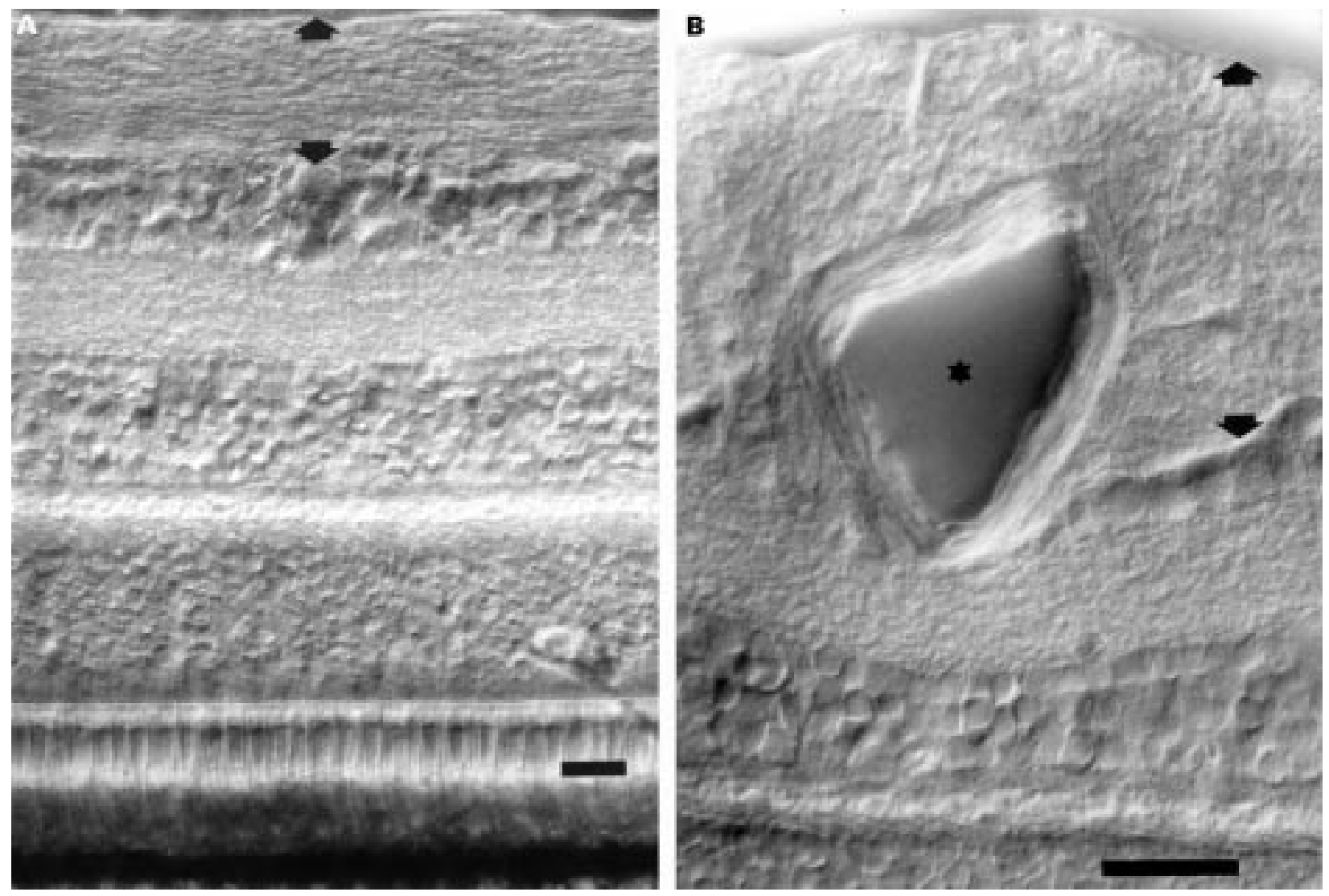

Figure 2 Photomicrographs showing the retinal nerve fibre layer as seen with Normarski optics. (A) At the edge of the macula, the retinal layers can clearly be seen. Scale bar $50 \mu \mathrm{m}$. (B) At the disc margin large vessels are seen within the nerve fibre layer. The nerve fibre layer retinal ganglion cell interface is shown (arrow). Scale bar $50 \mu \mathrm{m}$

\section{Results}

The retardation image for the eye in which the nerve fibre layer thickness measurements were made is shown in Figure 1A and was of similar quality to those obtained clinically. Brighter areas on the retardation map correspond to regions of increased retardation as seen at the inferior and superior poles of the optic disc. Blood vessels and the optic disc itself (Fig 1B) are clearly demarcated as areas of lower retardation. The retinal sections in Figure 2 show the boundaries (arrowed) used to measure the nerve fibre layer; these could be easily defined using DIC optics, avoiding the need for a counterstain and the artefacts that result from tissue dehydration. Figure $2 \mathrm{~B}$ shows the increased thickness of the nerve fibre layer at the disc margin, illustrating how retinal blood vessels run deep in the nerve fibre layer as they approach the optic disc. Nerve fibre layer measurements overlying these blood vessels were excluded from the analysis. One section was analysed independently by two observers to estimate any measurement bias. ${ }^{18}$ For 23 independent measurements of nerve fibre layer thickness along the section, the mean difference between observers was $8.6 \mu \mathrm{m}$ (SD 7.6 $\mu \mathrm{m})$.

Figure 3 shows selected nerve fibre layer thickness measurements that illustrate the changes in nerve fibre layer thickness around the disc. The histological location of blood vessels used to align and rotate the maps have been superimposed on the location taken from the digital map to illustrate the correspondence between landmarks. The disc margins at the superior and inferior poles are also shown (open arrows). The nerve fibre layer was thickest at the temporal aspects of the superior and inferior disc poles, peaking at $210 \mu \mathrm{m}$ superiorly and $286 \mu \mathrm{m}$ inferiorly (Fig 3 ). At the nasal and temporal aspects, the thickness reduced to $114 \mu \mathrm{m}$ and $100 \mu \mathrm{m}$ respectively. Superiorly

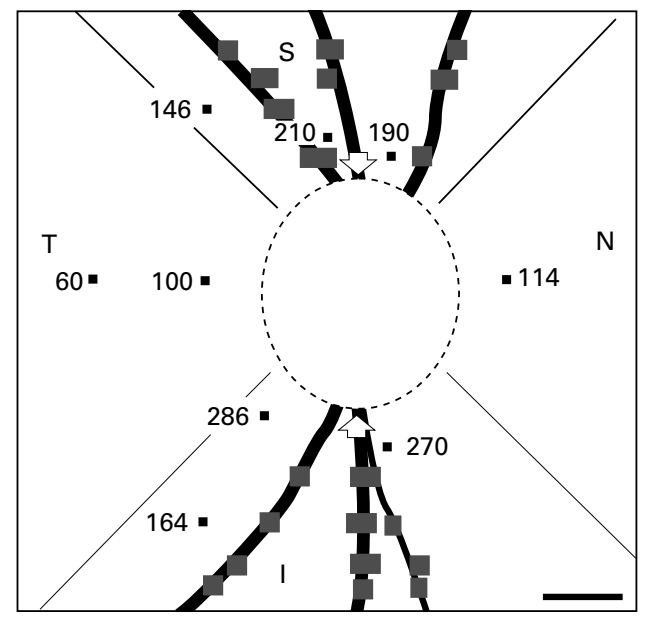

Figure 3 Diagram showing representative retinal nerve fibre layer thickness measurements $(\mu \mathrm{m})$ with respect to the disc margin (interrupted line). Major blood vessels are shaded in black and correspond to the field of view in Figure 1. Peripapillary retina is divided into superior $(S)$, inferior (I), temporal (T), nasal (N) sectors by lines that intersect at 90 degrees at the centre of the optic disc. Scale bar 2 degrees (495 $\mu \mathrm{m}$ ). 

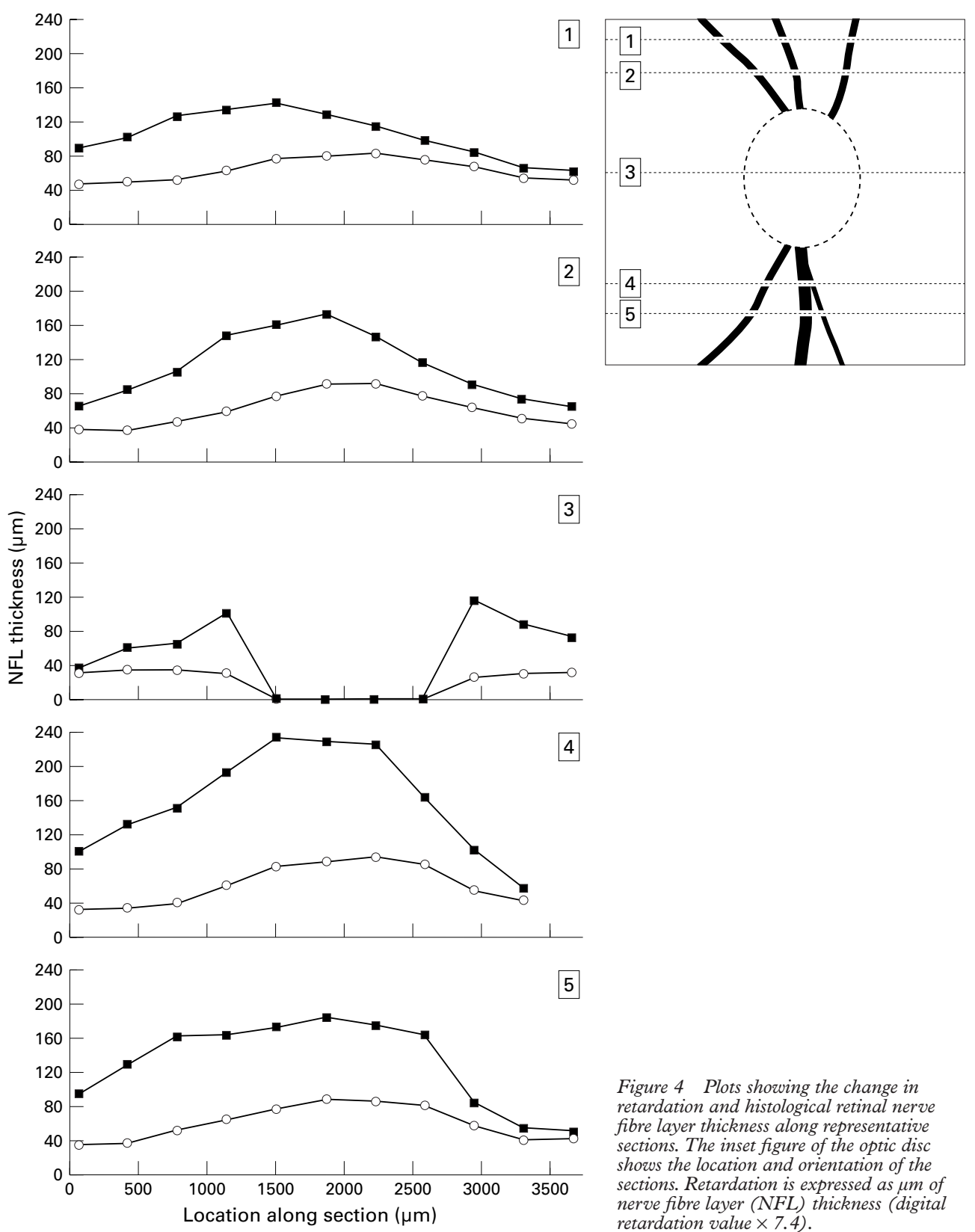

Figure 4 Plots showing the change in retardation and histological retinal nerve fibre layer thickness along representative sections. The inset figure of the optic disc shows the location and orientation of the sections. Retardation is expressed as $\mu m$ of nerve fibre layer (NFL) thickness (digital retardation value $\times 7.4$ ).

and inferiorly, axon bundles turned sharply towards temporal retina, thinning to approximately $20 \mu \mathrm{m}$ at the macula (not shown in Fig 3 ). The relation between retardation and thickness is illustrated for five representative sections in Figure 4. Retardation values are given in $\mu \mathrm{m}$ (as read off the digital map provided by the NFA) and have not been converted to degrees of retardation.

Satisfactory histology retardation matches were obtained at 216 locations and are plotted in Figure 5. The overall correlation coefficient for nerve fibre layer thickness against retardation at these locations was $r=0.70(\mathrm{p}<0.001)$. More complex curve fitting of the same data using quadratic and cubic models gave only a modest improvement in the correlation coefficient (to 0.72 in both cases).

Regional estimates for this correlation were calculated by dividing the image into four 90 degree quadrants (superior, inferior, nasal, and

temporal) centred upon the optic as shown in Figure 3. The correlation coefficients, gradients, and intercepts for these segments are given in Table 1 . The highest correlation is seen at the inferior disc pole, with less positive correlations occurring superiorly and temporally. The lowest correlation was seen at the

Table 1 Summary of correlation coefficients ( $r$ ) for retinal nerve fibre layer thickness (histological measurements) against retardation value for matched retinal locations. The definitions of the superior, inferior, nasal, and temporal sectors are described in Figure 3

\begin{tabular}{llllll}
\hline & Total & Temporal & Superior & Nasal & Inferior \\
\hline $\mathrm{N}$ & 216 & 86 & 26 & 59 & 45 \\
$r$ & 0.7 & 0.48 & 0.52 & 0.062 & 0.76 \\
$\mathrm{~b}$ & 14.02 & 22.61 & 10.75 & 1.61 & 20.85 \\
$\mathrm{~b} 0$ & 15.82 & -19.91 & 29.41 & 71.67 & -41.86 \\
$\mathrm{p}$ & $<0.001$ & $<0.001$ & 0.007 & 0.64 & $<0.001$ \\
\hline
\end{tabular}

$\mathrm{N}=$ number of pairs used to calculate the correlation coefficient in each sector. $\mathrm{b}=$ gradient of the straight line fitted to the data $(\mu \mathrm{m} /$ degree $) . \mathrm{b} 0=$ intercept on the $\mathrm{y}$ axis (thickness, $\mu \mathrm{m})$. 


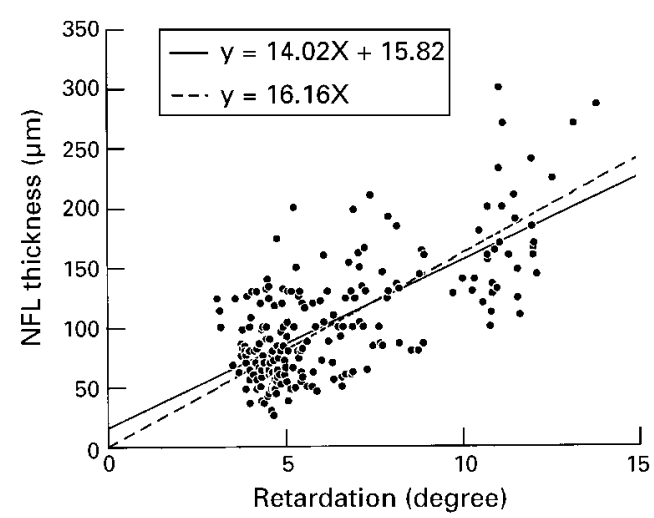

Figure 5 Plot of retardation value against measured nerve fibre layer (NFL) thickness for all thickness retardation pairs. $n=216 . r=0.70, p=<0.001$. The equation describing the straight line fits is shown inset. The broken line shows the straight line fit through the origin.

nasal aspect of the disc. The slope of the line (b) also varied around the disc, being steeper in the inferior and temporal aspects and shallowest nasally.

Figure 6 shows how retardation and nerve fibre layer thickness change around the optic disc. Histology retardation comparisons were obtained at 47 points located in a peripapillary zone with inner and outer boundaries at 1.5 and 2.0 disc diameters from the disc centre. Note that, inferiorly, there is better alignment between peak values for retardation and thickness compared with the superior disc pole where the peak retardation value lies slightly nasal to the peak nerve fibre layer thickness.

To provide comparison with clinically obtained retardation images, the corresponding mean retardation profiles for 10 normal right and left eyes are shown in Figure 7 (mean age 63.8 (SD 8.6) years, five male, five female). Retardation values were taken from a peripapillary zone as described for Figure 6. The profiles have been averaged for left and right eyes

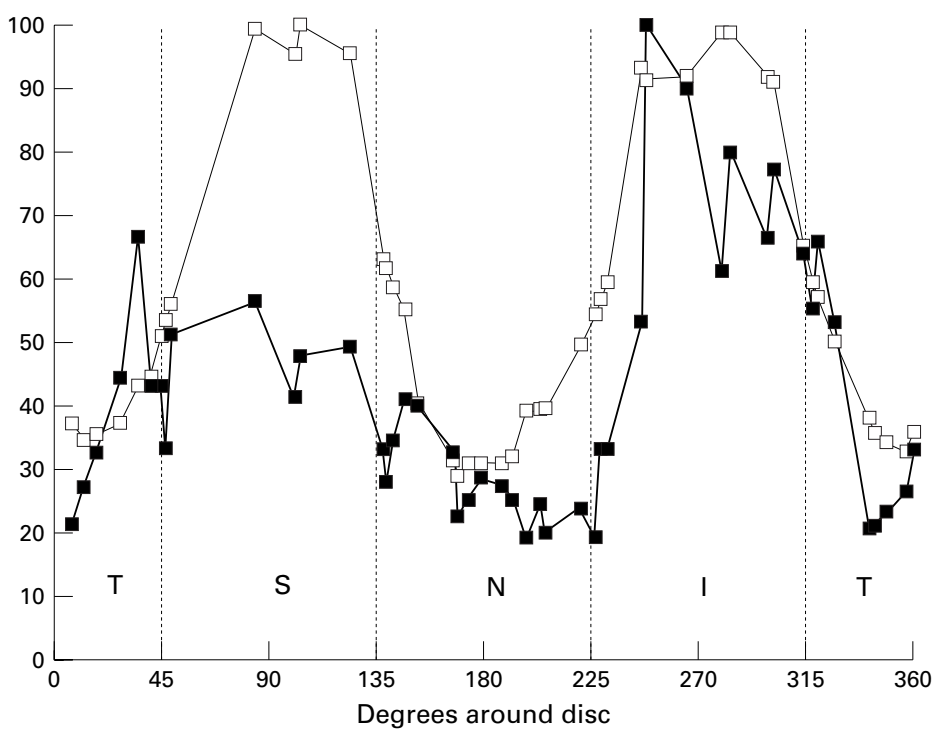

Figure 6 Plot of retardation and corresponding nerve fibre layer (NFL) thickness measures for points lying within a 1.5-2.0 disc diameter zone (from the disc centre) around the optic disc. The temporal aspect of the disc lies at 0 degrees and the superior aspect at 90 degrees. Bold line, solid markers: nerve fibre layer thickness. Fine line, open markers; retardation. Retardation and thickness scales have been normalised relative to their peak values to facilitate comparison. and are normalised with respect to their peak values. As seen in the primate, the inferior peak retardation values, which tended to have greater amplitude, were closely aligned with the inferior pole of the optic disc. Superiorly, the peak retardation values occurred on the nasal aspect of the superior disc pole.

\section{Discussion}

The present study describes the relation between nerve fibre layer thickness with retardation measures obtained in vivo in an intact primate eye. It extends previous work ${ }^{14}$ comparing histological measurement of the retinal nerve fibre layer thickness with the retardation values and provides sufficient data to explore any regional variation in the correlation between these variables. Our histological assessment of retinal nerve fibre layer thickness in the primate is in broad agreement with previous studies ${ }^{514}$ and with clinical descriptions of the contour of the peripapillary retinal nerve fibre layer. ${ }^{19-21}$ We found the peak nerve fibre layer thickness to be slightly greater than reported previously for the primate. For example, Radius ${ }^{22}$ reported mean nerve fibre layer thicknesses of $228 \mu \mathrm{m}$ and $194 \mu \mathrm{m}$ at the superior and inferior disc poles. Ogden ${ }^{23}$ reported detailed analysis in a single retina that showed a thickness of $200 \mu \mathrm{m}$ approximately $1 \mathrm{~mm}$ inferior to the disc and $200 \mu \mathrm{m}$ at the superior disc border. The difference between our data was greater at the nasal and temporal aspects where the thickness was no more than $50 \mu \mathrm{m}$ in both these studies compared with our study in which the thickness did not fall below $100 \mu \mathrm{m}$. These discrepancies may reflect tissue processing artefacts since in previous reports the retinal tissue was dehydrated and stained before analysis.

The nerve fibre trajectory from the optic disc margin is consistent with earlier descriptions of the retinal nerve fibre layer in the primate ${ }^{5}$ and the human. ${ }^{24} 25$

When all the histology and retardation pairs are treated together, nerve fibre layer retardation shows a good correlation $(r=0.70)$ with retinal nerve fibre layer thickness. However, it is lower than reported in an earlier study of nerve fibre layer polarimetry in the primate ${ }^{14}(r$ $=0.83$ ). Several factors may account for this difference. Most importantly, in this study the eye was imaged in vivo, with the cornea in place. The correlation, therefore, not only reflects the device's ability to measure nerve fibre layer retardation, but also its capacity to neutralise the confounding effect of corneal birefringence. For the previous anatomical study, the Fourier ellipsometer used a shorter wavelength laser and provided a more complete measure of the retardation produced by the nerve fibre layer. By contrast, the NFA is restricted to measuring changes in linear birefringence $^{14}{ }^{16}$ possibly accounting for the difference in the range of retardation values for the two studies. Since different illuminating wavelengths were used in these studies and the same birefringence will have different values at different wavelengths, the retardation is better 

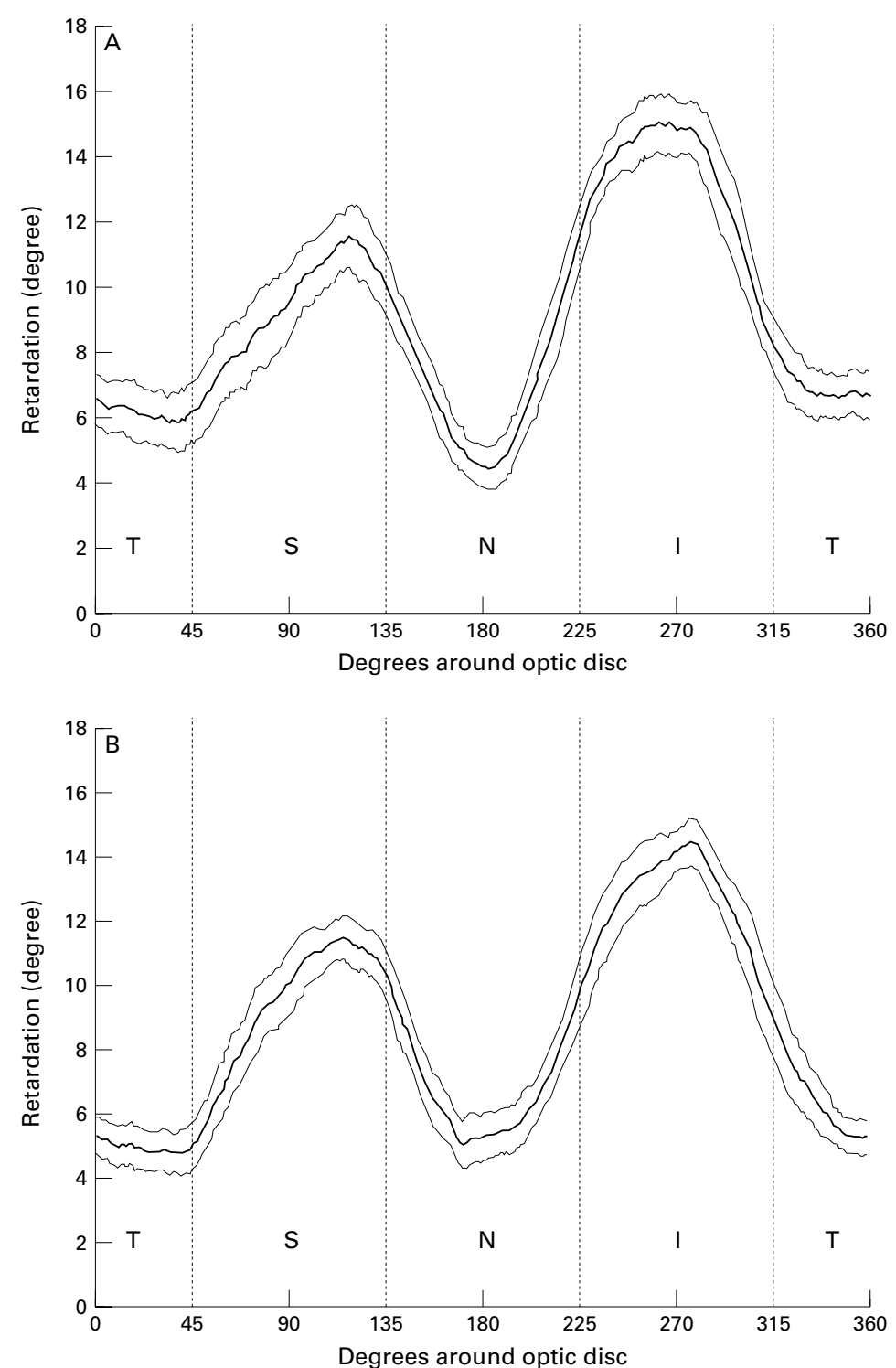

Figure 7 Plot of retinal nerve fibre layer (RNFL) around the optic disc for 10 normal (non-glaucomatous) patients. Bold line, mean RNFL. Fine lines show the $95 \%$ confidence intervals. (A) right eyes, (B) left eyes.

compared in terms of pathlength difference $\Gamma$ $(\mathrm{nm})$ rather than degrees. The units are related by the following function ${ }^{26}$ :

$$
\Gamma=\delta_{\operatorname{deg}} \star \lambda / 360
$$

where $\Gamma=$ retardation (pathlength difference, $\mathrm{nm}), \delta_{\mathrm{deg}}=$ retardation in degrees, and $\lambda$ is the wavelength of the illuminating beam for the retardation measurement $\delta_{\mathrm{deg}}$.

Thus, with nerve fibre layer thickness in the range $20-214 \mu \mathrm{m}$, retardation values ranged from 1.3 to $33.8 \mathrm{~nm}(0.9-23.7$ degrees $)$ in the study by Weinreb et $a l^{14}$ compared with 6.7-26.2 nm (3.1-12.1 degrees) for the present study. When the constant is removed from the equation describing the relation between the nerve fibre layer and retardation, we found that one degree of retardation is equivalent to $16.2 \mu \mathrm{m}$ of nerve fibre layer compared with $7.4 \mu \mathrm{m}$ reported previously. ${ }^{14}$ The difference is less marked when account is taken of the different illuminating wavelengths with these values corresponding to $5.2 \mu \mathrm{m} / \mathrm{nm}$ for
Weinreb et al compared with $7.5 \mu \mathrm{m} / \mathrm{nm}$ for the present study.

The peripapillary variation in the correlation coefficient and linear relation for retardation and nerve fibre layer thickness has not previously been described. It is possible that it results from poor alignment of the retardation and thickness maps. We think this is unlikely in view of the ease with which landmarks could be aligned in the two maps (less than one degree of relative rotation was required). The extent of histological artefact can be estimated by calculating the relation between the linear dimensions of the retinal map and the angular dimensions of the retardation map (retinal magnification factor). Based on the thickness of each section, the retinal segment covered $3655 \mu \mathrm{m}$ vertically and $3709 \mu \mathrm{m}$ horizontally. The retardation map subtended 15 degrees giving a vertical retinal magnification factor of $243.7 \mu \mathrm{m} / \mathrm{deg}$ and horizontal magnification factor of $247.3 \mu \mathrm{m} / \mathrm{deg}$. Both values are close to the previously reported retinal magnification factor in the macaque at the fovea of 223 $\mu \mathrm{m} / \mathrm{deg},{ }^{27}$ suggesting little tissue distortion.

At the superior and inferior disc poles some variation in correlation might be anticipated from inspection of the scans from the 10 normal patients. Superiorly, the peak retardation values lie slightly nasal to the superior pole whereas the inferior retardation peak is aligned to the inferior disc pole. A similar picture is seen in the primate (Fig 6), in which the peak retardation value at the superior pole lies slightly nasal to the peak nerve fibre layer thickness. Interestingly, this alignment can also be seen in studies in which the cornea had been removed prior to scanning (see Dreher et al fig $4^{10}$ ), suggesting that although corneal birefringence may account for some of this discrepancy, it is not the sole explanatory factor. We do not have an explanation for the nasal shift in the superior retardation value.

In spite of the complexities of the nerve fibre layer retardation relation shown in the present study, the NFA has significant clinical potential. Retardation measurements are consistent with the known properties of the retinal nerve fibre layer in normal and glaucomatous eyes ${ }^{16}$ and show an age related decreas $\mathrm{e}^{28}$ that would be expected from histological studies of the optic nerve..$^{29}{ }^{30} \mathrm{~A}$ recent large study using the device to discriminate between normal and glaucomatous eyes reported very high sensitivity and specificity. ${ }^{31}$ However, in order to be clinically useful, retardation values at the superior and inferior disc poles had to be expressed relative to each other or to the nasal aspect of the disc. ${ }^{32}$ Quantitative comparison of retinal nerve fibre layer photography and the NFA in the assessment of glaucomatous and ocular hypertensive patients showed, at best, a correlation coefficient of $0.53 .^{33}$ Indeed, a stronger correlation of mean defect was found with scored nerve fibre layer photographs than with polarimetric measurements. ${ }^{33}$

Histological comparison of retinal nerve fibre layer thickness with retinal nerve fibre layer polarimetry has not been undertaken in the human. The importance of such an 
analysis is emphasised by the recent description of the peripapillary human retinal nerve fibre layer thickness ${ }^{34}$ which showed a smaller modulation around the optic disc compared with the primate. Nasal retinal nerve fibre layer (RNFL) was only slightly reduced compared with superior retina while temporal RNFL was approximately $100 \mu \mathrm{m}$ thinner than superior RNFL. The peripapillary modulation in retardation obtained with the NFA for the human is more marked than would be expected and merits further investigation.

In conclusion, we have confirmed the correlation of retinal nerve fibre layer thickness with retinal nerve fibre retardation measures. We have also demonstrated regional variability in this correlation around the optic disc that may affect the clinical efficacy of this technology for the diagnosis of changes in nerve fibre layer thickness. It is important to note that the data in this study are based on a single image in a single animal. Further studies analysing the variables that influence the in vivo nerve fibre layer retardation correlation are required before the NFA can be considered for routine clinical use.

Proprietary interest: None

We thank Dr Klaus Reiter of Laser Diagnostic Technologies for his technical assistance with the NFA and the reviewers for helpful comments.

1 Sommer A, Katz J, Quigley H. Clinically detectable nerve fiber layer atrophy precedes the onset of glaucomatous field fiber layer atrophy precedes the onset of
loss. Acta Ophthalmol 1991;109:77-83.

2 Tuulonen A, Airaksinen P. Initial glaucomatous optic disk and retinal nerve fiber layer abnormalities and their and retinal nerve fiber layer abnormalities
progression. Am f Ophthalmol 1991;109:77-83.

3 Quigley H, Dunkelburger G, Green W. Studies of retinal ganglion cell atrophy correlated with automated perimetry in human eyes with glaucoma. Am F Ophthalmol 1989;107: 453-64.

4 Sommer A, Quigley H, Robin A, et al. Evaluation of nerve fiber layer assessment. Arch Ophthalmol 1984;102:1766-71.

5 Quigley H, Addicks E. Quantitative studies of retinal nerve fiber layer defects. Arch Ophthalmol 1982;100:807-14.

6 Niessen A, van den Berg T, Langerhorst C, et al. Grading of retinal nerve fiber layer with a photographic reference set. Am f Ophthalmol 1995;120:577-86.

7 Quigley H, Reacher M, Katz J, et al. Quantitative grading of nerve fiber layer photographs. Ophthalmology 1993;100. 1800-7.

8 Zinser G, Harbarth U, Schroder H. Formation and analysis of three dimensional data with the laser tomographic scanof three dimensional data with the laser tomographic scan-
ner. In: Nasemann J, Burk R, eds. Scanning laser ophthalmoscopy and topography. Munich: Quintessenz, ophthalm.
1990.

9 Weinreb R, Dreher A, Bille J. Quantitative assessment of the optic nerve head with the laser tomographic scanner. Int $\mathscr{f}$ Ophthalmol 1989;13:25-9.
10 Dreher A, Reiter K, Weinreb R. Spatially resolved birefringence of the retinal nerve fiber layer assessed with a retinal laser ellipsometer. Appl Optics 1992;31:3730-5.

11 Dreher A, Reiter K. Retinal laser ellipsometry: a new method for measuring the retinal nerve fiber layer thickness distribution. Clin Vis Sci 1992;7:481-8.

12 Hemenger R. Birefringence of a mdeium of tenuous parallel cylinders. Appl Optics 1989;28:4030-4.

13 Zhou Q, Knighton R. Light scattering and form birefringence of parallel cylindrical arrays that represent cellular organelles of the retinal nerve fiber layer. Appl Optics 1997; 36:2273-85.

14 Weinreb RN, Dreher AW, Coleman A, et al. Histopathologic validation of Fourier-ellipsometry measurements of retinal nerve fiber layer thickness. Arch Ophthalmol 1990;108:55760.

15 Van Blokland G, Virhelst S. Corneal polarization in the living human eye explained with a biaxial model. f Opt Soc Am A 1987;4:82-90.

16 Weinreb R, Shakiba S, Zangwill L. Scanning laser polarimetry to measure the nerve fiber layer of normal and glaucomatous eyes. Am f Ophthalmol 1995;119:627-36.

17 Laser Diagnostic Technologies. NFA Hardware and Software Manual. Supplement Version 1.6x. San Diego: LDT, 1994.

18 Altman D. Practical statistics for medical research. London: Chapman and Hall, 1991.

19 Caprioli J, Ortiz-Colberg R, Miller JM, et al. Measurements of peripapillary nerve fiber layer contour in glaucoma. $\mathrm{Am}$ f Ophthalmol 1989;108:404-13.

20 Caprioli J, Miller JM. Measurement of relative nerve fiber layer surface height in glaucoma. Ophthalmology 1989;96: 633-9.

21 Caprioli J. The contour of the juxtapapillary nerve fiber layer in glaucoma. Ophthalmology 1990;97:358-65.

22 Radius R. Thickness of the retinal nerve fiber layer in primate eyes. Arch Ophthalmol 1980;98:1625-9.

23 Ogden T. Nerve fiber layer thickness of the primate retina: thickness and glial content. Vis Res 1983;23:581-7.

24 Airaksinen P, Drance S, Douglas G, et al. Visual field and retinal nerve fibre layer comparisons in glaucoma. Arch Ophthalmol 1985;103:205-7.

25 Jonas J, Nguyen N, Naumann G. The retinal nerve fiber layer in normal eyes. Ophthalmology 1989;96:627-32.

26 Shurcliff W. Polarized light. London: Oxford University Press, 1962

27 Perry V, Cowey A. The ganglion cell and cone distributions in the monkey's retina: implications for central magnification factors. Vis Res 1985;25:1795-810.

28 Poinooswamy D, Fontana L, Wu J, et al. Variation of nerve fibre layer thickness measurements with age and ethnicity by scanning laser polarimetry. Br f Ophthalmol 1997;81: $350-4$.

29 Dolman C, McCormick A, Drance S. Ageing and the optic nerve. Arch Ophthalmol 1980;98:2053-8.

30 Balazi A, Rootman J, Drance S, et al. The effect of age on the nerve fibre population of the optic nerve. Am $\mathcal{F}$ Ophthalmol 1984;97:760-6.

31 Tjon-Fo-Sang M, Lemij H. The sensitivity and specificity of nerve fiber layer measurements in glaucoma as determined with scanning laser polarimetry. Am f Ophthalmol 1997; 123:62-9.

32 Tjon-Fo-Sang M, De Vries J, Lemij H. Measurement by nerve fiber analyzer of retinal nerve fiber layer thickness in normal subjects and patients with ocular hypertension. Am f Ophthalmol 1996;122:220-7.

33 Niessen A, Van Den Berg T, Langerhorst C, et al. Retinal nerve fiber layer assessment by scanning laser polarimetry and standardized photography. Am f Ophthalmol 1996;121: 484-93.

34 Varma R, Skaf M, Barron E. Retinal nerve fiber layer thickness in normal human eyes. Ophthalmology 1997;103:
2114-19. 